Distilled water $\quad 110 \mathrm{~mL}$

Alcohol, $95 \% \quad 780 \mathrm{~mL}$

Glacial acetic acid $\quad 4 \mathrm{~mL}$

Combine and stir until dyes are dissolved. Immediately ready for use.

Store at room temperature. Shelf life at least 2 years. Filter before use.

Staining time: 15 seconds to 2 minutes.

Note: To prevent dye crystals from precipitating from the solution, rinse slides in $70 \%$ alcohol prior to eosin stain. If the precipitate appears on the slides, rinse slides in absolute alcohol with a level of alcohol higher than that of the eosin stain. This can be accomplished with both manual or automatic staining.

$\begin{array}{ll}\text { *Picro-Eosin } 3: & \\ \text { Eosin Y } & 1.6 \mathrm{gm} \\ 80 \% \text { alcohol } & 720 \mathrm{~mL} \\ \text { Saturated picric acid } & 80 \mathrm{~mL}\end{array}$

Dissolve eosin in alcohol: add picric acid solution. Mix well. Filter

References:

1. Preece, A. 1972. A Manual for Histologic Technicians. Little, Brown and Co., Boston.

2. Feldman, A. Anatech Ltd., Battle Creek, MI., personal communication. 3.

Prophet, E. 1992. Laboratory Methods in Histotechnology Armed Forces Institute of Pathology, American Registry of Pathology, Washington. D.C.

Cheryl Crowder, Louisiana State University

\section{Freeze-Drying Fungal Hyphae}

I've done some preliminary work using Leica's freeze-drying unit on fungal material. These were prepared to maintain chemical integrity. I've freezedried Bergamot (Monard didyma) leaves with a powdery mildew fungal infection and yeast (Saccaromyces cerevisae). The leaves came out fine but the fungal hyphae were collapsed. The yeast came out very nicely. I ran both fixed (Karnovsky's) and unfixed pieces. All were cryo prepared by propane plunge freezing. All were examined at $2.0 \mathrm{kV}$ uncoated. Overall I was pleased with the results, I haven't had a chance to go over all of the samples but the chemical fixation seemed to be better for maintaining the fungus. I would run both unfixed and fixed as I believe the mechanical agitation of adding a liquid solution may have washed away some fungal material. Aldehyde and/or Osmium vapor fixation would likely be less disruptive, and preliminary results look promising.

Freeze-drier time and temperature schedule was:

48 hours @ $-80^{\circ} \mathrm{C}$

36 hours @-60 C

24 hours @ $-40^{\circ} \mathrm{C}$

12 hours @ $-30^{\circ} \mathrm{C}$

12 hours @- $15^{\circ} \mathrm{C}$

24 hours @- $50^{\circ} \mathrm{C}$

6 hours @+100 C

30 hours @ $+20^{\circ} \mathrm{C}$

Vacuum was maintained with a cryosorption pump.

Images are on my web page. The unfixed yeast pics are there already. Follow the links from Research Projects to Sample prep for SEM...

http:/www. personal.psu.edu/ejb11

Edward J. Basgall, The Pennsylvania State University

A Simple And Cheap Holder

For Staining Several TEM Grids At A Time

Take ring-shaped slices of BEEM capsules $4-5 \mathrm{~mm}$ high, cut evenly spaced perpendicular slits along one side of the ring, leaving room on the other side for pinching the ring which opens the slits.

Then place the grids into the slits, grabbing just the rim of the grid. The grids are held in place when the rings are released.

It takes a bit of practice to get the slits spaced right and to learn how to get several grids into the slits without losing the first ones you put in.

Stain using $10 \mathrm{~mL}$ beakers, submersing the grid rings in the stain, and rinse by bobbing the rings up and down in water, holding the rings with tweezers. Excess water can be blotted from between the wet grids with points of filter paper.

This takes more stain, but I think the results are cleaner. Sometimes I produce a "lucky" ring that will hold 6 giids for me!

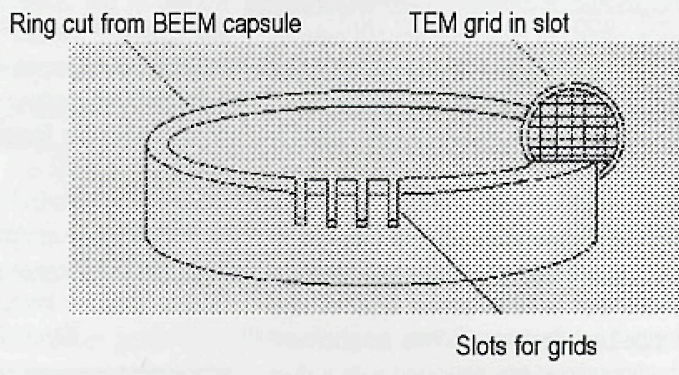

Note: slot and grid placement shown is for drawing clarity only Julie Gross, University of Connecticut Health Center
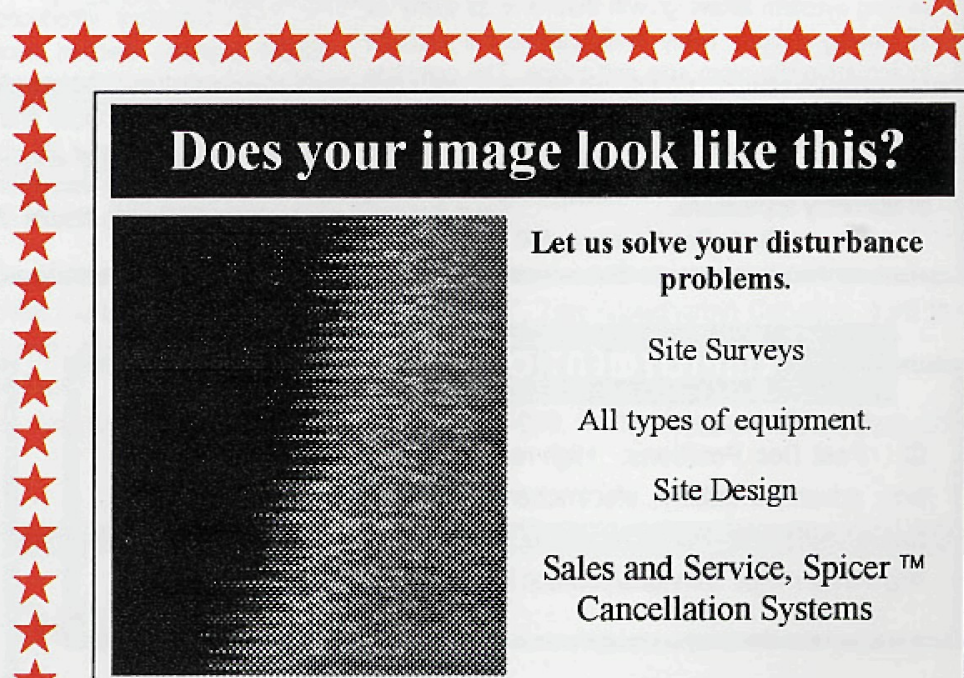

Let us solve your disturbance problems.

Site Surveys

All types of equipment.

Site Design

Sales and Service, Spicer TM Cancellation Systems

ELIMINATE

- Vibration

- Fields (EMI)

- Acoustics

- $\mathrm{AC}$ Power and grounding problems 